\title{
Prozesstaugliche Plasmadiagnostik auf Basis der Multipol-Resonanz-Sonde
}

Dipl.-Ing. Jens Oberrath ${ }^{1}$, Dipl.-Ing. Christian Schulz ${ }^{2}$, Dipl.-Ing. Robert Storch ${ }^{3}$, Dipl.-Ing. Tim Styrnoll ${ }^{4}$, Dr.-Ing. Martin Lapke ${ }^{1}$, Priv.-Doz. Dr.-Ing. Thomas Mussenbrock ${ }^{1}$, Prof. Dr.-Ing. Peter Awakowicz ${ }^{4}$, Prof. Dr. Ralf Peter Brinkmann ${ }^{1}$, Prof. Dr.-Ing. Thomas Musch ${ }^{3}$, Prof. Dr.-Ing. Ilona Rolfes ${ }^{2}$

Anschrift(en):

${ }^{1}$ Lehrstuhl für Theoretische Elektrotechnik, 0234/32-26336

${ }^{2}$ Lehrstuhl für Hochfrequenzsysteme, 0234/32-27383

${ }^{3}$ Lehrstuhl für Elektronische Schaltungstechnik, 0234/32-27115

${ }^{4}$ Lehrstuhl für Allgemeine Elektro- und Plasmatechnik, 0234/32-22487

Fakultät für Elektro- und Informationstechnik, Ruhr-Universität Bochum, D-44780 Bochum

oberrath@tet.rub.de, christian.schulz-hfs@rub.de, robert.storch@est.rub.de, styrnoll@aept.rub.de, lapke@tet.rub.de, thomas.mussenbrock@rub.de, awakowicz@aept.rub.de, brinkmann@tet.rub.de, thomas.musch@rub.de, ilona.rolfes@rub.de

\section{Industriekompatible Plasmadiagnostik}

Die Überwachung und zielgerichtete Beeinflussung technischer Plasmen erfordert die messtechnische Bestimmung ihrer inneren Zustandsgrößen, vor allem der Elektronendichte und der mittleren Elektronenenergie. Der Begriff „Plasmadiagnostik“ umfasst alle Einrichtungen und Verfahren, die für eine solche Messung der Zustandsgrößen eines Plasmas herangezogen werden können. Von den bekannten Methoden sind jedoch nur wenige für den Einsatz in einem industriellen Umfeld geeignet. Eine industriekompatible Plasmadiagnostik muss robust und stabil sein, soll eine eindeutige und kalibrationsfreie Auswertung ermöglichen, und darf den zu überwachenden Prozess weder elektrisch noch chemisch stören. Im Hinblick auf Anschaffung, Unterhalt und Platzbedarf müssen die Kosten vertretbar sein. Im Fall schichtabscheidender Plasmaprozesse sind diese Forderungen besonders schwer zu erfüllen. Nahezu alle bekannten Diagnostiken scheiden als ungeeignet aus: Optisch-spektroskopische Verfahren (Optical Emission Spectroscopy, Optical Absorption Spectroscopy) sind zu aufwendig und liefern nur indirekte Aussagen; Massenspektroskopie scheitert an dem unzureichenden Zugang (sowie an den Kosten), konventionelle elektrische Diagnostik (mittels Langmuir-Sonden) wird durch die abgeschiedenen dünnen Schichten erheblich verfälscht. Auch das Plasmametrologieverfahren SEERS (Self Excited Electron Resonance Spectroscopy) ist nicht geeignet, da es eine Hochfrequenzanregung der Plasmen voraussetzt.

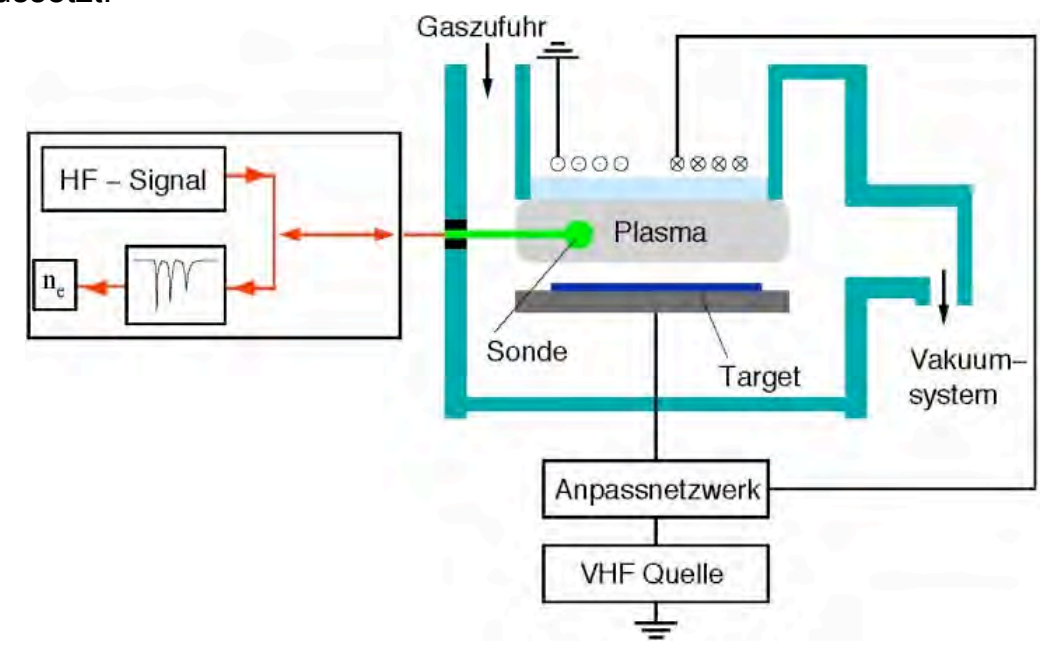

Abb. 1: Das Grundkonzept der aktiven Resonanzspektroskopie 
Ein attraktives Konzept für eine industriekompatible Diagnostik schichtabscheidender Plasmaprozesse ist hingegen die in Abb. 1 schematisch dargestellte aktive Resonanzspektroskopie. Dieses Messkonzept beruht auf der universalen Eigenschaft aller Niederdruckplasmen, bei Anregung mit einem Hochfrequenzsignal in Nähe der Elektronenplasmafrequenz $\omega_{p}$ in ausgeprägte Resonanz zu geraten. Ist durch die spektrale Analyse der gemessenen Systemantwort $S(\omega)$ die Plasmafrequenz bekannt, so kann die Elektronendichte leicht formelmäßig bestimmt werden.

In der Vergangenheit wurden bereits verschiedene Realisierungen des Grundprinzips der aktiven Resonanzspektroskopie vorgeschlagen. (Eine Übersicht findet sich in Ref. [1].) Unter diesen Vorschlägen befinden sich die Plasma Absorption Probe [2], die Wave Cut-Off Probe [3], die Plasma Transmission Probe [4], und die Hairpin Probe [5]. Diese Konzepte wurden zwar experimentell verifiziert, konnten sich in der technisch-industriellen Praxis jedoch nicht durchsetzen, und zwar aufgrund folgender prinzipbedingter Nachteile:

- In allen bisherigen Konzepten sind die im Plasma angeregten Resonanzmoden von derart komplizierter Struktur, dass der Zusammenhang zwischen dem beobachteten Spektrum und der zu bestimmenden Elektronendichte formelmäßig nicht darstellbar und oft auch nicht eindeutig ist. Damit ist keine exakte Analyse des Signals möglich, sondern es müssen empirische Formeln (Näherungsverfahren) angewendet werden. Die Auswertung des Signals und damit die Messung selbst erhalten so einen teilweise nicht unbeträchtlichen Modellfehler (Siehe Analyse in [6,7].)

- Die bisherige technische Realisierung der Auswertung war zu aufwendig und für den technischen Einsatz deutlich zu teuer. Zur Erzeugung des Hochfrequenzsignals und zur Analyse des gemessenen Resonanzspektrums wurden kommerzielle heterodyne Netzwerkanalysatoren verwendet. Diese sind jedoch als Universal-Messinstrumente konzipiert und damit nicht nur für Regelungszwecke viel zu langsam, sondern auch für einen industriellen Einsatz zu kostenaufwendig.

\section{Das Prinzip der Multipol-Resonanz-Sonde}

Die Multipol-Resonanz-Sonde (Multipole Resonance Probe $=$ MRP) ist eine innovative Realisierung des skizzierten Grundkonzeptes der aktiven Plasmaresonanzspektroskopie. Eine der Innovationen besteht aus einer neuen symmetrischen Gestaltung der in das Plasma einzubringenden Sonde. Dieses neuartige Sondendesign besitzt sowohl elektrisch wie geometrisch eine hohe Symmetrie (siehe Abb. 2.) Der Kopf der Sonde besteht aus einer dielektrisch umhüllten Anordnung von zwei voneinander isolierten Halbkugeln, die jeweils mit den Polen einer symmetrischen, auch als Halterung dienenden HochfrequenzZuleitung verbunden sind. Durch das HF-Signal wird das umgebende Plasma zur Resonanz angeregt.

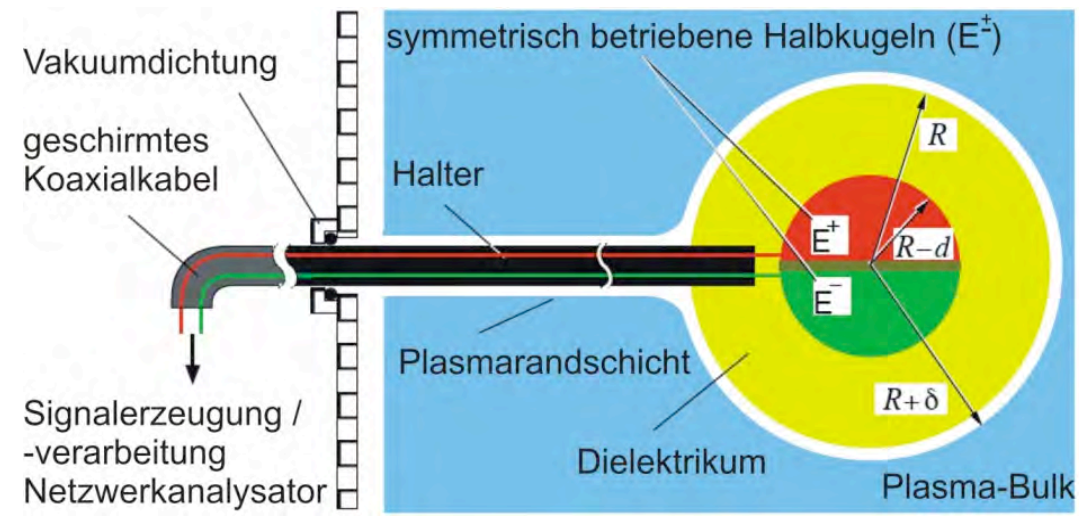

Abb. 2: Schema der Multipol-Resonanz-Sonde: Dargestellt ist ein Schnitt durch die Sonde 
Aufgrund des Aufbaus kann die Sonde und ihre Wechselwirkung mit dem Plasma weitgehend analytisch beschrieben werden. Die angeregten Resonanzen liegen unterhalb der Elektronenplasmafrequenz $\omega_{p e}$ aber deutlich höher als die lonenplasmafrequenz ${ }^{\omega_{p i}}$. Daraus folgt, dass die lonen zu schwer sind dem elektrischen Feld zu folgen. Es kann daher angenommen werden, dass im Plasma ein reiner ElektronenLeitungsstrom sowie ein Verschiebungsstrom fließen. Benutzt wird die „kalte Plasma-Approximation“, die sowohl Elektronenträgheit als auch Reibung durch Neutralteilchenstöße beinhaltet. Es konnte gezeigt werden, dass die elektrostatische Näherung gerechtfertigt ist, d.h., dass das Elektrische Feld durch ein Potential $\phi$ beschrieben werden kann. Berücksichtigt man all diese Näherungen kann unter Vernachlässigung der Zuleitung (numerische elektromagnetische und elektrostatische Untersuchungen unterstützen diese Annahme) das elektrostatische Potential aufgrund der durch die Bauform gegebenen radialen Symmetrie als Lösung der Poisson-Gleichung angegeben werden [8].

$$
\phi^{i}(r, \vartheta)=\sum_{s=1}^{\infty}\left(A_{s}^{i} r^{2 s-1}+B_{s}^{i} r^{-2 s}\right) P_{2 s-1}(\cos \vartheta)
$$

Die unbekannten Koeffizienten $A_{s}^{i}$ und $B_{s}^{i}$ bezeichnen hierbei die Multipolkoeffizienten im jeweilig ${ }^{i}$-ten Medium (Dielektrikum, Randschicht und Plasma). Sie ergeben sich nach Entwicklung der Randbedingung auf der Sondenoberfläche in Kugelflächenfunktionen als Lösung eines linearen Gleichungsystems.

Letztendlich kann zum Beschreiben des Resonanzverhaltens der Sonde der Strom

$$
I=\int_{E^{+}} j \omega \epsilon_{0} \epsilon_{D} \mathbf{\nabla} \phi \cdot d^{2} \vec{r}
$$

durch eine Elektrodenfläche berechnet werden (Der Strom durch die zweite Elektrode ist aufgrund der Symmetrie genau ${ }^{-I}$ ). Das Ergebnis kann in eine Admittanz (dargestellt in Abb. 3)

$$
Y=\frac{I}{V}=j \omega C_{v a c}+\sum_{s=1}^{\infty}\left(\frac{1}{j \omega C^{s}}+\frac{j \omega+v_{\theta}}{\eta_{s}^{2} C_{s} \omega_{p_{\theta}}^{2}}\right)^{-1}
$$

umgerechnet werden. $C_{\text {vac }}$ beschreibt hier die kapazitive Kopplung, die auch ohne Plasma herrscht, $v_{e}$ beschreibt eine effektive Stoßfrequenz. Die Parameter $C_{s}$ und $\eta_{s}$ sind längliche Funktionen der Sondenparameter, Radius ${ }^{R}$, Dielektrikumsdicke ${ }^{d}$, Permittivität ${ }_{\downarrow} D$ sowie der Randschichtweite $\delta$.

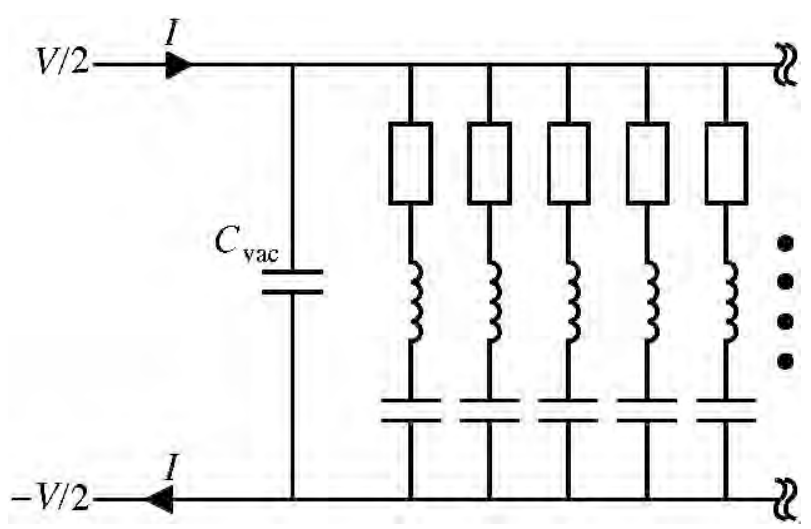

Abb. 3: Ersatzschaltbild des Systems Sonde/Plasma

Wie Admittanz oder Ersatzschaltbild zeigen, kann die Systemantwort $S(\omega)$ als eine Überlagerung 
unendlich vieler Resonanzmoden aufgefasst werden. Deren charakteristische Parameter (Resonanzfrequenz und Dämpfung) sind jeweils einfache algebraische Funktionen der gesuchten Plasmaparameter (Elektronendichte und Stoßfrequenz).

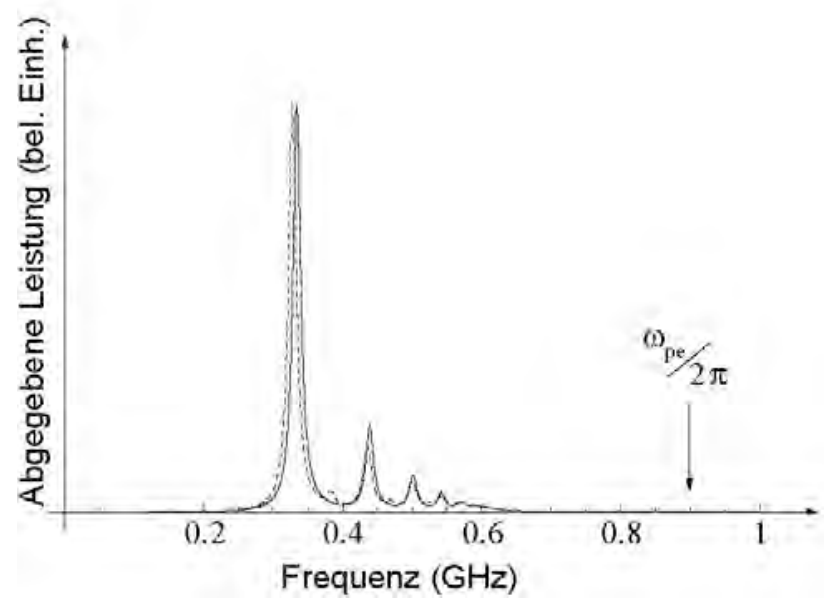

Abb. 4: Numerisch berechnete Systemantwort einer MRP mit Halter und HF-Zuführung (gestrichelt) im Vergleich zu der analytisch berechneten Systemantwort einer idealisierten MRP (durchgezogen). Die Maxima der Systemantwort $S(\omega)$ entsprechen den namensgebenden „Multipol-Resonanzen“, wobei das erste und stärkste Maximum der Dipolresonanz entspricht. (Details in [8].)

\section{Die Mess- und Auswertelektronik}

Eine weitere Innovation des gesamten Messsystems der Multipol-Resonanz-Sonde stellt die Mess- und Auswerteelektronik dar. Zur Einbettung der Sonde in ein wirtschaftliches Gesamtsystem zur Prozessdiagnose ist es unabdingbar, für die Erzeugung und Auswertung des hochfrequenten Signals eine spezialisierte Elektronik zu entwickeln. Um die Einsatzfähigkeit der MRP in einer vielzahl unterschiedlicher Plasmen zu gewährleisten, muss die Elektronik in der Lage sein, ein Signal mit einer Bandbreite von $200 \mathrm{MHz}-6 \mathrm{GHz}$ zu erzeugen und dieses mit einer möglichst hohen Messwiederholrate auswerten zu können. Dies erweitert das Spektrum der diagnostizierbaren Plasmen auf die gepulsten Plasmen. Zur Realisierung einer solchen Elektronik wird ein Verfahren gewählt, welches an die Zeitbereichsreflektometriesysteme angelehnt und dessen Blockschaltbild in Abb. 5 angegeben ist.

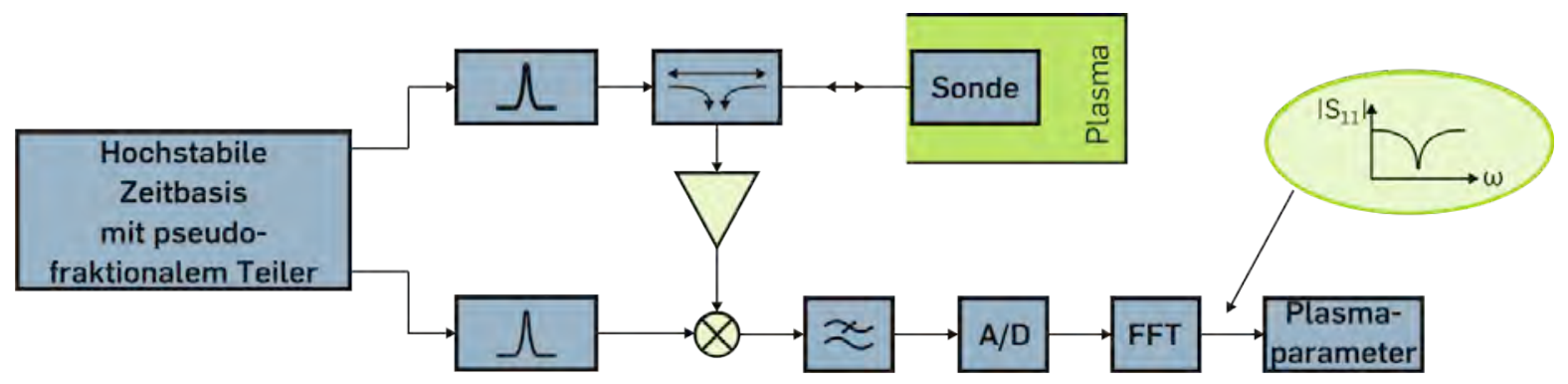

Abb. 5: Blockschaltbild der Ansteuer- und Auswerteelektronik

Hierbei wird ein extrem kurzer elektromagnetischer Puls generiert, der eine Pulsbreite von ca. 100 ps aufweist (s. Abb. 6 links). Entsprechend der Fouriertransformation ergibt sich so die geforderte Bandbreite von ca. $6 \mathrm{GHz}$ (s. Abb. 6 rechts). 

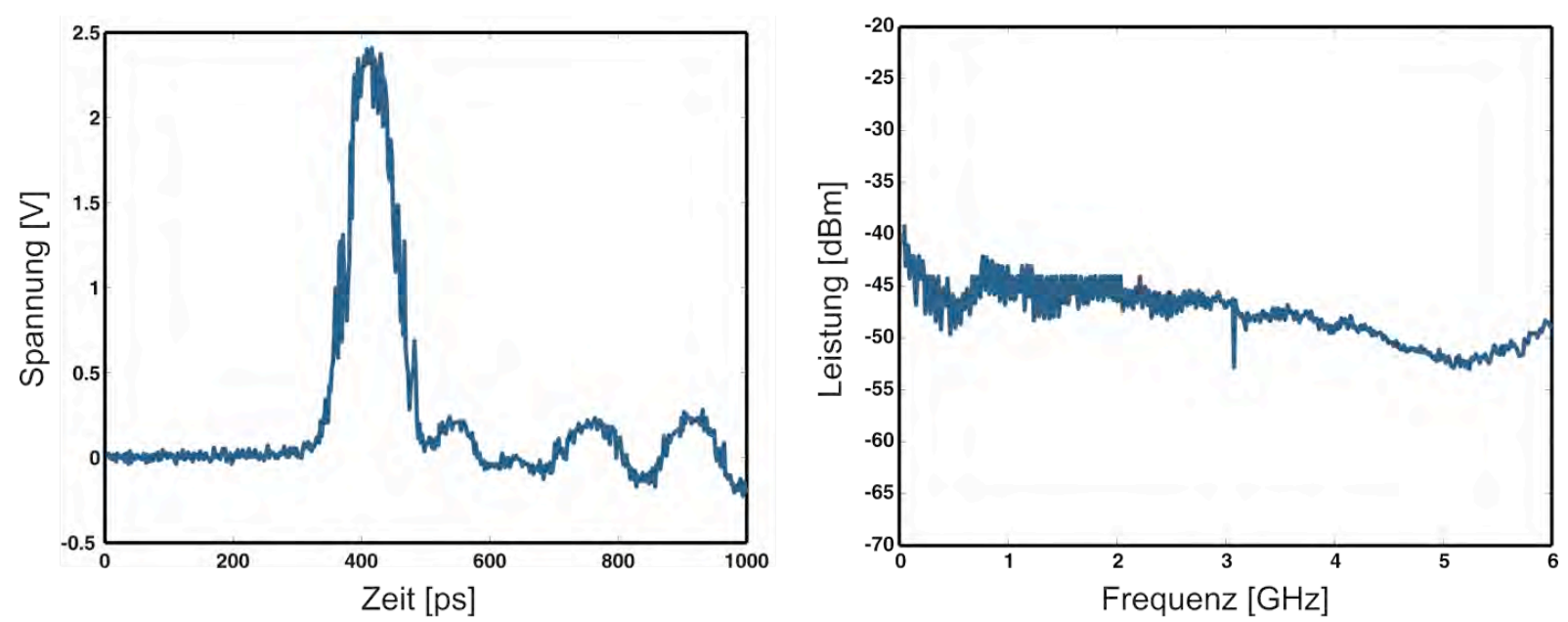

Abb. 6: Puls im Zeit- (links) und Frequenzbereich (rechts)

Dieser Puls wird nun über die MRP in das Plasma eingespeist und von diesem reflektiert. Für eine Auswertung des reflektierten Pulses ist eine Digitalisierung notwendig. Dazu wird sein Spektrum mit Hilfe einer Unterabtastung auf einige 10 bis $100 \mathrm{kHz}$ gestaucht. Der dafür benötigte zweite Pulsgenerator sollte für eine optimale Leistungsausbeute exakt die gleichen Eigenschaften aufweisen wie der Erste. Beide Pulsgeneratoren werden von einer hochstabilen Zeitbasis mit einer minimalen Differenzfrequenz angesteuert. Hierbei bestimmt die Pulsfolgefrequenz die spektrale Auflösung der Messung und die Differenzfrequenz die Messwiederholrate. Der im Frequenzbereich gestauchte Puls wird mit Hilfe eines 14 Bit Analog-Digital-Wandlers digitalisiert und die Daten können über die USB 2.0 Highspeed Schnittstelle mit einer Datenrate von ca. $30 \mathrm{MB} / \mathrm{s}$ an einen PC übertragen werden. Das Spektrum des digitalisierten Puls kann nun im Bezug auf die Parameter des Plasmas ausgewertet werden.

Die Kernkomponenten des Systems sind der Pulsgenerator, sowie die hochstabile Zeitbasis. Der Pulsgenerator basiert auf einer sogenannten Step-Recovery-Diode, welche auf Grund eines speziellen Dotierprofils eine extrem kurze Transitzeit der Ladungsträger aufweist. Die so erzielbare schnelle Stromänderung wird mit Hilfe zweier Induktivitäten in einen differentiellen Spannungspuls überführt, wodurch die Ansteuerung des nachfolgenden Mischers optimiert wird. Die hochstabile Zeitbasis darf als Resultat der Pulsbreite von ca. 100 ps lediglich einen zeitlichen Jitter im Bereich weniger Pikosekunden aufweisen. Um dies zu realisieren, kommt ein neuartiges Konzept zum Einsatz, welches eine extrem phasenrauscharme Quarzreferenzfrequenz mit Hilfe von programmierbaren Frequenzteilern herunterteilt. Zur Generierung genügend kleiner Differenzfrequenzen werden diese Frequenzteiler periodisch umprogrammiert und das Ausgangssignal schmalbandig gefiltert um die Programmierfrequenz zu unterdrücken. Die Zeitbasis erlaubt so Differenzfrequenzen von unter $100 \mathrm{~Hz}$ bei einer Pulswiederholfrequenz von $10,7 \mathrm{MHz}$ mit einem zeitlichen Jitter von etwa 1 ps [9].

\section{Realisierung eines Demonstrators}

Um das Konzept der Multipol-Resonanz-Sonde in der Praxis umzusetzen und die Praxistauglichkeit zu überprüfen, wurde an der Ruhr-Universität Bochum in Zusammenarbeit der oben genannten Lehrstühle ein Demonstrator (Abb. 7) entwickelt. Wichtig bei der Entwicklung war zum einen die Gewährleistung der elektrischen Symmetrie, die für die Auswertung der Messung über das Modell unabdingbar ist, und zum anderen ein stabiler Betrieb der Sonde bei hohen Frequenzen. 


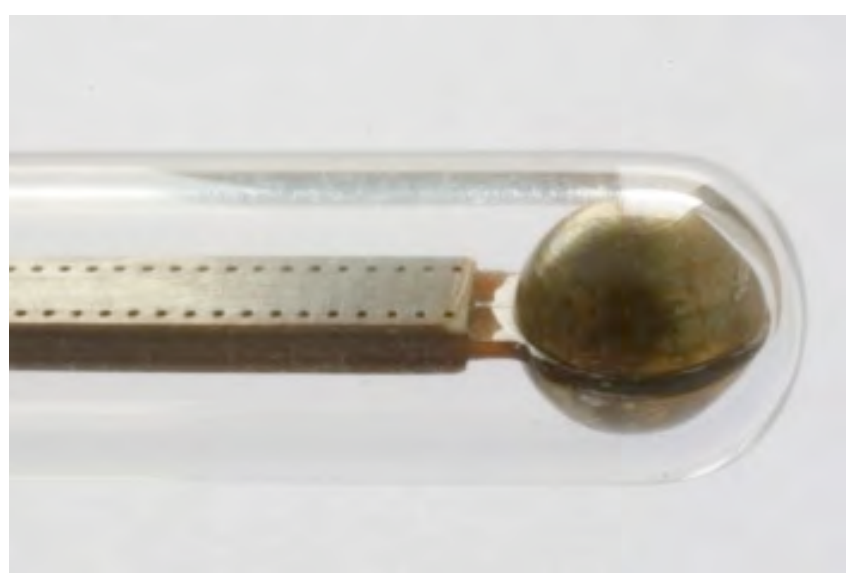

Abb. 7: Foto des Demonstrators der MRP bestehend aus Sondenkopf, Symmetrierglied und Ummantelung

Die Gewährleistung der elektrischen Symmetrie könnte mit einem 4-Tor Netzwerkanalysator sichergestellt werden, jedoch widersprechen die hohen Anschaffungskosten der industriekompatiblen Umsetzung der MRP. Alternativ wäre ein individueller Signalgenerator denkbar, der ein symmetrisches Hochfrequenzsignal erzeugt, das aber unmöglich über eine Zuleitung symmetrisch zur Sonde übertragen werden kann, weil schon leichte Verbiegungen des Kabels unterschiedliche Siganllaufzeiten hervorrufen. Daher bietet es sich an, das Signal unsymmetrisch bis zur Sondenhalterung zu führen und dort die Symmetrierung mit Hilfe eines Symmetrierglieds zu realisieren. Offensichtlich ist die geometrische Symmetrie durch die Halterung bei der realen Sonde nicht mehr gegeben, aber Abb. 4 zeigt, dass der Fehler bei der Auswertung vernachlässigbar ist.

Mit der Entscheidung das Symmetrierglied in die Halterung der Sonde zu integrieren, muss dieses so konzipiert sein, dass es sowohl die Symmetrierung als auch die Anpassung des Wellenwiderstandes zwischen Koaxialkabel und Sondenkopf realisiert. Zur Entwicklung des Symmetriergliedes und zur weiteren Optimierung ist eine Simulation des Hochfrequenzverhaltens der gesamten Sonde nötig. Eine solche Simulation kann mit Hilfe des Programms CST-Microwave Studio ${ }^{\circledR}$ durchgeführt werden. Nach ausführlicher Analyse konnte die optimale Struktur des Symmetriegliedes ermittelt werden [10].

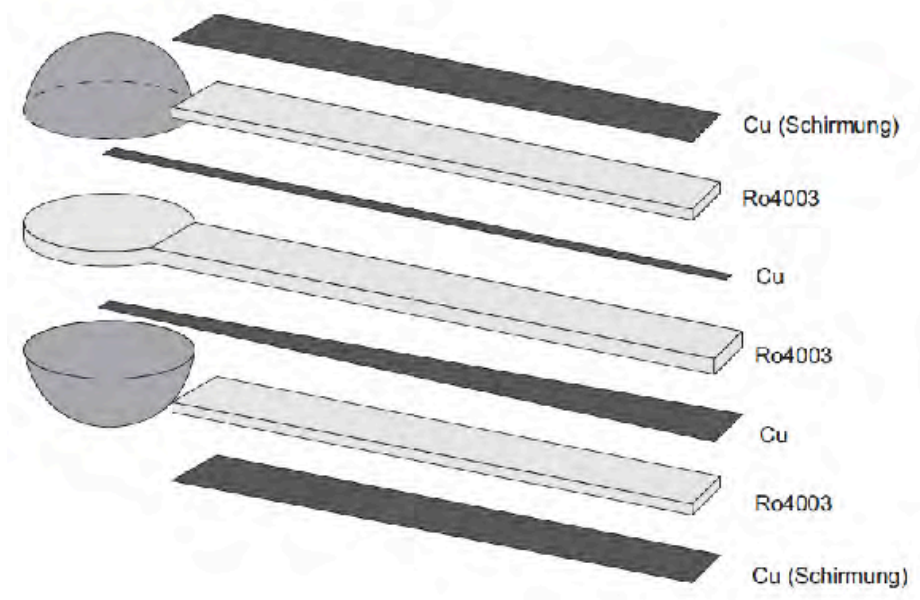

Abb. 8: Explosionszeichnung des Sondensystems ohne dielektrische Ummantelung 
Abb. 8 zeigt eine Explosionszeichnung des Sondensystems ohne dielektrische Ummantelung. Die dunklen Lagen bestehen aus Kupfer und dienen zum einen als Schirmung (oben und unten) und zum anderen als Signallagen (mitte). Die grauen Schichten sind spezielle Isolatoren $\left(\mathrm{RO} 4003^{\circledR}\right)$, die besonders gut für hochfrequente Systeme geeignet sind. Die Verbindungsschichten, die sogenannten Prepregschichten, wurden in der Skizze vernachlässigt, müssen aber in der Simulation wegen ihrer dielektrischen Eigenschaft berücksichtigt werden. Die Elektroden selbst sind aus Kupfer gefertigt. In der Skizze erkennt man deutlich, dass eine der Kupferlagen zum Sondenkopf hin verjüngt ist. Durch eine derartige Verjüngung wird das ankommende unsymmetrische Signal zum Sondenkopf symmetriert und zusätzlich der Wellenwiderstand angepasst. Die Simulationen ergaben bei einem Elektrodenradius von $R=4 \mathrm{~mm}$ mit einer Dicke des Dielektrikums von $d=1 \mathrm{~mm}$ und einer Permittivität von $\epsilon_{r}=4,6$, der $200 \mu \mathrm{m}$-dicken $\mathrm{RO} 4003^{\circledR}$-Lagen und den $17 \mu \mathrm{m}$-dicken Kupfersignallagen, dass die untere Signallage von $2 \mathrm{~mm}$ auf $0,5 \mathrm{~mm}$ verjüngt und die obere von $0,3 \mathrm{~mm}$ auf $0,5 \mathrm{~mm}$ verbreitert werden muss um optimale Symmetrie zu erhalten. Die gesamte Länge des Symmetriergliedes beträgt $26 \mathrm{~mm}$. Eine detailierte Beschreibung des Demonstrators befindet sich in [11].

\section{Messungen mit der Multipol-Resonanz-Sonde}

Nach Fertigstellung des Demonstrators wurden erste Messungen in einem DICP (Double Inductive Coupled Plasma) in Argon-, Wasserstoff-, Stickstoff- und Sauerstoff-Gemischen durchgeführt und mit einem etablierten Diagnostiksystem, dem Langmuirsondensystem (APS3) [12], verglichen. Wie in Abb. 9 zu sehen ist, ermöglichen zwei gegenüberliegende Flansche am DICP-Reaktor, APS3 und MRP in einer Ebene zu positionieren. Dies gewährleistet eine zeitsynchrone Messung des identischen Plasmazustandes im zylindersymmetrischen Reaktor mit beiden Diagnostiksystemen.

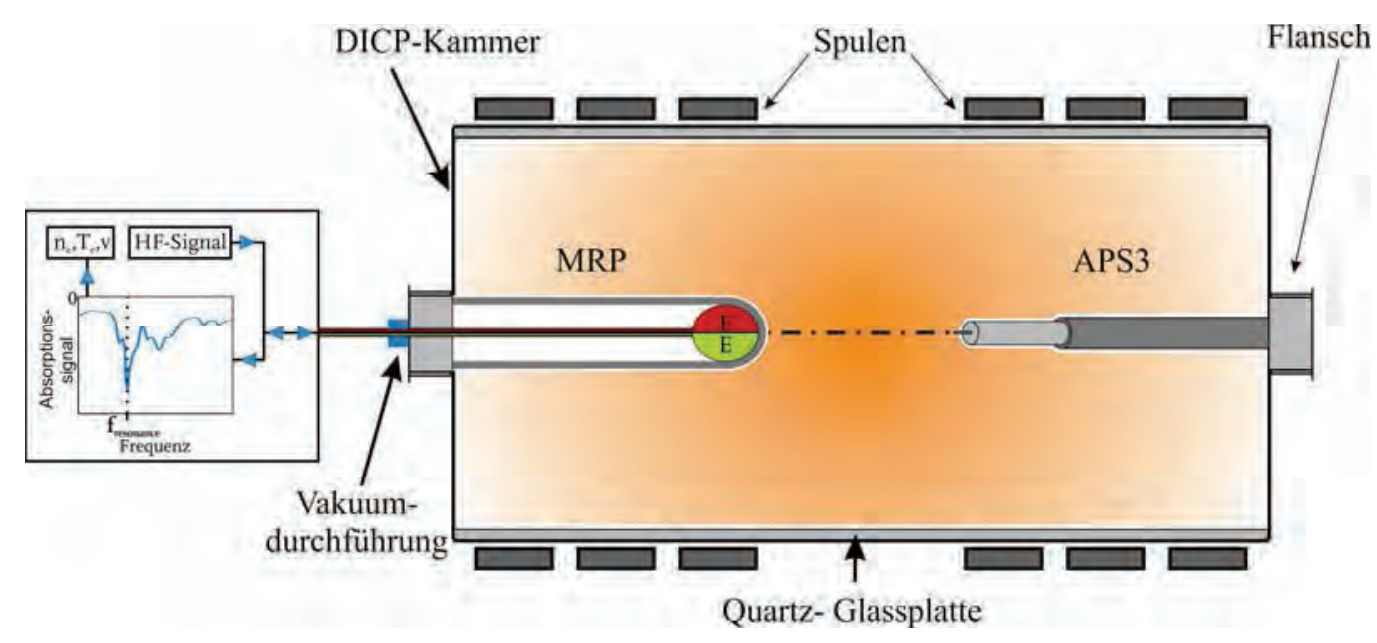

Abb. 9: DICP-Aufbau zur gleichzeitigen Messung mit MRP und Langmuir-Sonde

Im dargestellten DICP-Reaktor wurden Niederdruckentladungen betrieben und Gasgemische aus ArgonWasserstoff und Argon-Stickstoff mit einem Flussverhältnis von 100:5 sccm untersucht. Der Prozessdruck wurde im Bereich von $5 \mathrm{~Pa}$ bis $20 \mathrm{~Pa}$ variiert, die eingekoppelte Leistung von $250 \mathrm{~W}$ bis $750 \mathrm{~W}$. In Abb. 11 sind jeweils Leistungs- und Druckvariationen der beiden Gasgemische, gemessen im DICP, dargestellt. Man sieht eine sehr gute Übereinstimmung der Elektronendichte von Langmuirsonde zu Multipol-ResonanzSonde unabhängig von Leistung und Druck. 

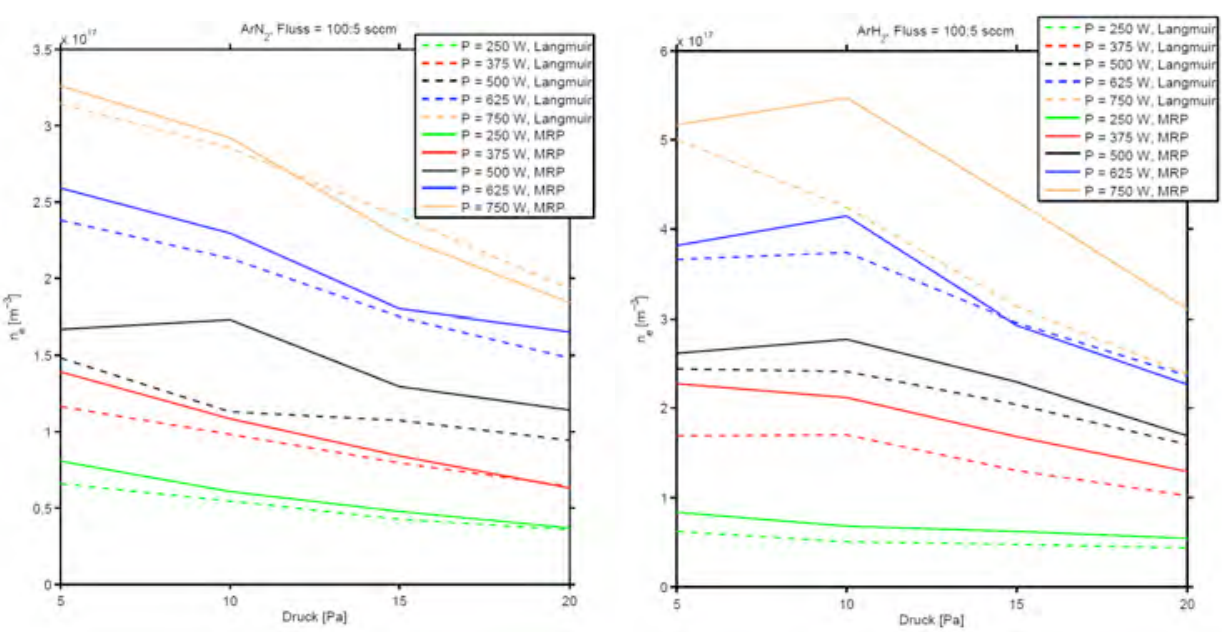

Abb. 10: Leistungs- und Druckvariation in einer Argon-Stickstoff (links) und Argon-Wasserstoff Entladung (rechts)

Um den Einfluss der Elektronen-Stoßfrequenz zu untersuchen, wurde eine Druckvariation durchgeführt. Wird der Druck bei konstanter Leistung variiert, so verändert man unweigerlich auch die Elektronendichte und somit die von der Multipol-Resonanz-Sonde gemessene Resonanzfrequenz. Aus diesem Grund wurde in einem Experiment die Leistung für jeden Druck so nachgeregelt, dass bei allen Drücken identische Elektronendichten und somit auch identische Resonanzfrequenzen gemessen wurden. Dies ermöglicht die eindeutige Untersuchung des Druckeinflusses auf das Absorptionsspektrum und ist in Abb. 11 (links) dargestellt. Man erkennt, dass mit steigendem Druck die Amplitude eines Absorptionspeaks kleiner und das Profil des Absorptionspeaks breiter wird, da aufgrund von mehr Elektron-NeutralteilchenStößen im Plasma die Dämpfung zunimmt.
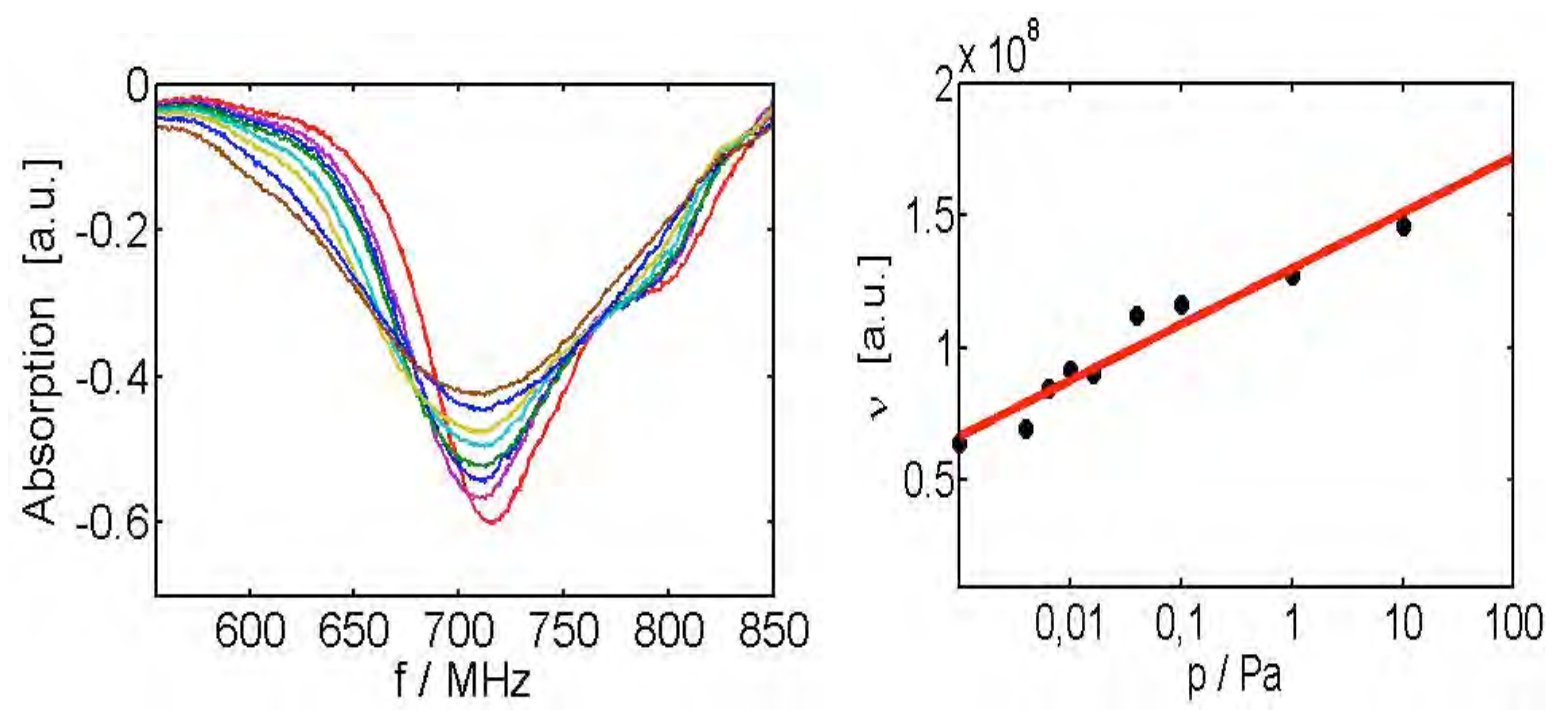

Abb. 11: Variation des Drucks bei konstanter Elektronendichte (links) und Stossfrequenz in Abhängigkeit des Prozessdrucks (rechts)

Werden, wie in Abb. 11 (rechts), die Stoßfrequenzen, die aus der Halbwertsbreite eines Absorptionspeaks berechnet werden können, gegen den Druck aufgetragen, so fällt auf, dass bei $p=0$ Pa die Stoßfreuquenz ${ }^{v}$ ungleich Null ist (Schnittpunkt mit der Ordinate in Abb. 12 rechts). Dies zeigt, dass sich die Stoßfrequenz nicht nur aus der Stoßfrequenz $v_{\varepsilon}$ für Stöße von Elektronen mit 
Neutralteilchen besteht, da diese bei verschwindendem Druck gegen Null gehen muss, sondern auch aus einer zweiten Stoßfrequenz, die mit $v_{k i n}$ bezeichnet wird. Diese zusätzliche Stoßfrequenz beschreibt den Einfluss des Feldes der MRP auf ein Elektron, welches eine Impulsänderung ohne Stoßpartner erfährt. Die gesamte Elektronen-Stoßfrequenz ergibt sich somit als Summe der beiden einzelnen. Hierbei kann $v_{e}$ aus bekannten Ratenkonstanten und bekanntem Prozessdruck errechnet werden. Der kinetische Anteil der Stoßfrequenz kann mit der „kalten Plasma-Approximation“, die der oben beschriebenen Theorie der Sonde zugrunde liegt, nicht erklärt werden. Zur genauen Beschreibung der zusätzlichen Stoßfrequenz wird ein kinetisches Modell der MRP benötigt.

Weitere Messungen in Plasmen mit unterschiedlicher Energieeinkopplung (CCP, MW, APS/Syrus Pro) untermauern die Funktionalität der MRP. Auch in dielektrisch beschichtenden Plasmen (in der Dünnschichtoptik und zur Herstellung von $\mathrm{SiO}_{x}$-Barriereschichten auf $\mathrm{PET}$ ) wurde die Robustheit der MRP in industriellen Prozessen erfolgreich überprüft [13].

\section{Zusammenfassung und Ausblick}

In diesem Beitrag wird die Multipol-Resonanz-Sonde vorgestellt und gezeigt, dass sie als prozesstaugliche Plasmadiagnostik geeignet ist.

Als erstes wird beschrieben, was unter einer prozesstauglichen Diagnostik bei Plasmaprozessen zu verstehen ist und das die aktive Resonanzspektroskopie ein vielversprechender Kandidat dafür ist. Das Konzept dieser Spektroskopie wurde in zahlreichen Bauformen umgesetzt, doch scheitern fast alle an den sehr strengen Bedingungen der Prozesstauglichkeit. Als besonders vielversprechende Bauform wird die MRP vorgestellt, die auf einem neuartigen Sondendesign beruht, welches die Herleitung eines eindeutigen Zusammenhangs zwischen Resonanzfrequenz und Elektronenplasmafrequenz erlaubt. Über diesen Zusammenhang kann die Elektronendichte des Plasmas lokal bestimmt werden. Eine eigens entworfene Mess- und Auswertelektronik verspricht eine kostengünstige Realisierung des gesamten Messsystems, das ansonsten mit Hilfe eines Netzwerkanalysators betrieben werden müsste. Wesentlicher Bestandteil der Sonde ist auch das Symmetrierglied als Teil der Sondenhalterung. Es sorgt für das benötigte symmetrische Signal am Sondenkopf und zusätzlich für die Anpassung des Wellenwiderstandes zwischen Koaxialkabel und Sonde.

Messungen mit der MRP wurden zum einem in einem DICP durchgeführt und direkt mit Messungen der Langmuir-Sonde verglichen. Es konnte gezeigt werden, dass die Ergebnisse der Messungen sehr gut übereinstimmen. Zum anderen wurden weitere Messungen in Plasmen mit unterschiedlicher Energieeinkopplung durchgeführt und damit erstens die Prozesstauglichkeit untermauert und zweitens der vielfältige Einsatzbereich der Sonde gezeigt. Bei einer speziellen Messung mit Druckvariation zeigte sich, dass die Stoßfrequenz der Elektronen bei verschwindendem Druck nicht gegen Null geht, sondern einen gewissen Offset aufweist. Dieses Phänomen kann nur mit Hilfe eines kinetischen Modells erklärt werden und ist Teil aktueller Forschung.

Zukünftig wird daran gearbeitet die MRP zu einem praxistauglichen Produkt weiter zu entwickeln. Dabei werden im Wesentlichen zwei Ansätze verfolgt:

Unter Verwendung eines bereits erprobten, vakuumgeeigneten Positionierungssystems kann die MRP zu einem ortsauflösenden System für die Plasmadiagnostik weiterentwickelt werden. Anwendungsgebiete sind die Entwicklung und Optimierung von Plasmaquellen und Prozessen, sowie die Übertragung von bereits entwickelten Prozessen auf neue Anlagen.

An geeigneter Stelle in einer Anlage fest montiert kann die MRP als stabiler Monitor zur Prozesskontrolle dienen. Überwacht werden können die Plasmakenngrößen Elektronendichte, -temperatur, und -stoßrate. Auf dieser Basis können Konzepte für AEC/APC (Advanced Equipment Control, Advanced Process 
Control) entwickelt werden. Auf der Basis eines stabilen Prozessmonitors können auch neue Konzepte zur Closed-Loop-Regelung von Plasmaprozessen entwickelt werden.

\section{Danksagung}

Diese Arbeit wurde unterstützt durch die DFG via Graduiertenkolleg 1051 und via Ruhr University Research School, sowie durch das BMBF-Projekt PluTO.

\section{Literatur}

[1] N. St. J. Braithwaite and R. N. Franklin, "Reflections on electrical probes", Plasma Sources Sci. Technol. 18014008 (2009)

[2] $\mathrm{H}$. Kokura et al., "Plasma absorption probe for measuring electron density in an environment soiled with processing plasmas", Jpn. J. Appl. Phys. 38 (1999)

[3] J.-H. Kim, S.-C. Choi, Y.-H. Shin, K.-H. Chung, "Wave cutoff method to measure absolute electron density in cold plasma", Rev. Sci. Instr., 75, 2706-2710 (2004)

[4] S. Dine et al., "A novel technique for plasma density measurement using surface-wave transmission spectra", Plasma Sources Sci. Technol. 14 (2005)

[5] R. Piejak et al., "Hairpin resonator probe measurements in RF plasmas", Plasma Sources Sci. Technol. 14 (2005)

[6] C. Scharwitz, M. Böke, S.-H. Hong, and J. Winter, "Experimental Characterisation of the Plasma Absorption Probe“, Plasma Proc. and Polymers, 4, 605-611 (2007)

[7] M. Lapke, T. Mussenbrock, R. P. Brinkmann, C. Scharwitz, M. Boeke, and J. Winter, "Modeling and simulation of the plasma absorption probe", Appl. Phys. Lett. 90, 121502 (2007)

[8] M. Lapke, T. Mussenbrock, and R. P. Brinkmann, "The Multipole Resonance Probe (MRP): A concept for simultaneous determination of plasma density, electron temperature, and collision rate in low-pressure plasmas", Appl. Phys. Lett. 93, 051502 (2008)

[9] R. Storch, T. Musch: "A highly stable timebase for subsampling systems using a fractional concept with short periodicity", Proceedings of the $25^{\text {th }}$ Asia-Pacific Microwave Conference, Melbourne, Australia (2011)

[10] C. Schulz, M. Lapke, J. Oberrath, R. Storch, T. Styrnoll, C. Zietz, P. Awakowicz, R. P. Brinkmann, T. Musch, T. Mussenbrock, I. Rolfes, "The Multipole Resonance Probe: Realization of an optimized radiofrequency plasma probe based on active plasma resonance spectroscopy”, MECAP 2010 (2010)

[11] M. Lapke, J. Oberrath, C. Schulz, R. Storch, T. Styrnoll, C. Zietz, P. Awakowicz, R. P. Brinkmann, T. Musch, T. Mussenbrock, I. Rolfes, "The Multipole Resonance Probe: Characterization of a Prototype", Plasma Sources Sci. Technol. 20 , 4, 042001 (2011)

[12] P. Scheubert et al., "Experimental and Theoretical Characterization of Inductively Coupled Plasma Source", J. Appl. Phys. 90, 587-98 (2001)

[13] T. Styrnoll et al., "Determintation of electron density and electron temperature using the Multipole Resonance Probe in technological plasmas", IX Workshop on Frontiers in Low Temperature Plasma Diagnostics (2011) 